\title{
LE DISTRICT EN ÉCHEC. L'EXEMPLE DES TANNERIES DE CHÂTEAU-RENAULT (V. 1860-1985)
}

\author{
par Cédric PERRIN
}

Avec sa concentration de tanneries, la petite ville de Château-Renault a longtemps été le premier pôle industriel de Touraine. Elle est un des nombreux districts industriels qui façonnent le paysage productif de la France des XIX ${ }^{\mathrm{e}}$ et $\mathrm{XX}^{\mathrm{e}}$ siècles. À son apogée, la cité regroupe près d'une vingtaine de ces établissements, sans compter les activités annexes qui caractérisent un district. Les cuirs castelrenaudins jouissaient d'une excellente réputation, en France et au-delà, au point d'être parfois copiés. Le concept de district industriel a connu un grand succès dans le champ des sciences économiques et sociales depuis la fin du $\mathrm{XX}^{\mathrm{e}}$ siècle $^{1}$. Issu des travaux de l'économiste britannique Alfred Marshall ${ }^{2}$, il est redécouvert dans les années soixante-dix par les chercheurs italiens, notamment par Giacomo Becattini, qui a enrichi la définition marshallienne de nouveaux critères ${ }^{3}$. Les districts italiens demeurent aujourd'hui un modèle d'alternative à la grande organisation, notamment fordiste ${ }^{4}$. Cependant, le modèle donne des signes d'épuisement. D'autres modèles d'organisation territoriale émergent, qui viennent relativiser la valeur canonique du district

1. Voir notamment Georges Benko, Alain Lipietz (dir.), Les régions qui gagnent. Districts et réseaux : les nouveaux paradigmes de la géographie économique, Paris, PUF, 1992. Michel Lescure (dir.), La mobilisation du territoire. Les districts industriels en Europe occidentale du XVII au XX ${ }^{e}$ siècle, Paris, CHEFF, 2006. Jean-François Eck, Michel Lescure (dir.), Villes et districts industriels en Europe occidentale $X V I I^{e}-X X^{e}$ siècles, Tours, Presses universitaires François-Rabelais, 2002. Jean-Claude Daumas, Pierre Lamard, Laurent Tissot (dir.), Les territoires de l'industrie en Europe (1750-2000), Entreprises, régulations et trajectoires, Besançon, Presses universitaires de Franche-Comté, 2007. Florent Le Bot, Cédric Perrin (dir.) Les chemins de l'industrialisation en Espagne et en France. Les PME et le développement des territoires (XVII ${ }^{e}-X X I^{e}$ siècles), Bruxelles, Peter Lang, 2011.

2. Alfred Marshall, Principles of Economics, Paris, Giard et Brière, 1906 ( $1^{\text {re }}$ éd. Londres, 1890), particulièrement les chapitres X et XI du livre 1. Disponible sur le site de l'université du Québec à Chicoutimi : http://classiques.uqac.ca/classiques/marshall_alfred/marshall_alfred.html.

3. Giacomo Becattini, « Dal settore industrial al distretto industriale : alla ricerca dellunitá d'indagine della economia industriale », Revista di economia e politica industriale, 1, 1979; du même auteur, en français : «Le district marshallien : une notion socio-économique », in Georges Benko, Alain Lipietz (dir.), Les régions qui gagnent..., op. cit. Arnaldo Bagnasco, Tre Italia. La problematica territoriale dello sviluppo italiano, Bologne, Il Mulino, 1977.

4. Par exemple, chez Suzanne Berger, Made in Monde. Les nouvelles frontières de l'économie mondiale, Paris, Le Seuil, 2006 (2005 pour l'édition originale en anglais). 
italien : le modèle du cluster de Michael Porter ${ }^{5}$, le district hub and spoke de la géographe Ann Markussen ${ }^{6}$... Intégrant ces nouveaux supports théoriques, les historiens économistes espagnols ont relativisé la place du modèle becattinien (ou néo-marshallien) au sein des districts espagnols ${ }^{7}$. Sur le plan méthodologique, Jean-Claude Daumas, par exemple, met en garde contre son aspect ahistorique en rappelant que les districts peuvent naître, se développer, décliner et mourir ${ }^{8}$. De fait, un nombre important de districts apparaissent aujourd'hui en difficulté.

Dans cette perspective, s'il n'est ni le plus important ni le plus connu, le district de Château-Renault présente un intérêt particulier. Il fournit un exemple précis de l'échec d'un district. Aujourd'hui, il n'en reste qu'un musée du cuir et quelques éléments de patrimoine industriel. La dernière tannerie a fermé ses portes en 1985. L'activité a totalement disparu. Qu'est-ce que cet échec montre des limites du district comme forme d'organisation de la production, et de son affaiblissement au $\mathrm{XX}^{\mathrm{e}}$ siècle ? Cette communication s'attachera à comprendre comment la dynamique de district, qui a fait le succès des industries castelrenaudines à un moment donné, s'est grippée et a fini par échouer. L'échec final doit être replacé dans le temps long, car au-delà de ses raisons immédiates, il prend ses racines, au moins partiellement, dans la période de réussite et d'apogée du district.

S'il a une influence évidente, le contexte de diminution des usages du cuir et de mondialisation ne peut suffire à l'expliquer. Les décisions des entrepreneurs doivent être examinées : attachement à certains choix techniques et commerciaux, à un type de produit, et à l'inverse mauvaises anticipations des évolutions de cette industrie et de ses marchés. À cet égard, la disparition du district se présente comme la somme des échecs individuels des entreprises qui l'ont composé. C'est aussi un échec du système lui-même. Le district apparaît ici comme une configuration efficace à une période donnée, mais qui présente aussi des faiblesses, voire des rigidités, et qui peine à s'adapter à l'évolution du capitalisme français. Après avoir fait la force de la petite cité tourangelle, l'organisation en district, et d'abord son ancrage territorial, se délite progressivement. Laissant de côté la phase de formation du district, l'article restitue le

5. Michael Porter, L'avantage concurrentiel des nations, Paris, Interéditions, 1993.

6. Ann Markusen, "Sticky places in Slippery space : a typology of industrials districts ", Economic geography, 72, 1996.

7. Jordi Catalan, José Antonio Miranda, Ramon Ramon-Muñoz (dir.), Distritos y clusters en la Europa del Sur, Madrid, LID, 2011. Jordi Catalan, « Distritos y clusters en la industrialización española. Una visión de largo plazo », in F. Le Bot et C. Perrin (dir.), Les chemins de l'industrialisation ..., op. cit.

8. Jean-Claude Daumas, « Districts industriels : du concept à l'histoire. Les termes du débat », Revue économique, 1, 2007. 
fonctionnement du district à son apogée (1860-1925), pour ensuite en montrer les faiblesses et enfin le lent déclin depuis l'entre-deux-guerres jusqu'à la disparition dans les années quatre-vingt.

\section{LE DISTRICT À SON APOGÉE : UN MONDE DE PME COOPÉRANTES}

Alfred Marshall a dégagé trois critères qui fondent classiquement la définition du district : la concentration d'entreprises d'un secteur avec ses industries annexes, un bassin de main-d'œuvre qualifiée, la circulation des techniques (évoquée dans la deuxième partie). Toutes ces caractéristiques se retrouvent sur le territoire de Château-Renault et contribuent donc à en faire un district. Les tanneries de la ville sont des PME qui, au-delà des relations de concurrence qui s'établissent entre entreprises d'une même branche d'activité agissant sur un même marché, coopèrent entre elles. Elles sont en « co-opétition ${ }^{9}$ ». Cette notion désigne des relations interentreprises bien particulières fondées tout à la fois sur la concurrence et la coopération. Elles ont été repérées notamment par les sociologues et économistes italiens dans les districts de la troisième Italie (localisée principalement en Émilie-Romagne, en Toscane, dans les Marches et en Vénétie, autour du quadrilatère Venise, Bologne, Florence, Rome). Les tanneurs castelrenaudins dépassent la concurrence qui les oppose pour s'organiser mutuellement et défendre l'identité collective de leur territoire. De la sorte, leur district peut aussi apparaître, tout au moins au XIX ${ }^{\mathrm{e}}$ siècle, comme un exemple de milieu innovateur ${ }^{10}$.

\section{A. UN TERRITOIRE DE PME}

Les tanneries de Château-Renault travaillent principalement de grandes peaux de bovins destinées à l'industrie de la chaussure, notamment à la fabrication des semelles. Au milieu du XIX ${ }^{\mathrm{e}}$ siècle, elles apparaissent clairement comme le principal pôle industriel de Touraine dans les rapports de la succursale de Tours de la Banque de France. Elles sont alors une dizaine (graphique 1) ${ }^{11}$. Leur

9. Adam Brandenburger, Barry Nalebuff, La co-opétition. Une révolution dans la manière de jouer concurrence et coopération, Paris, Village mondial, 1996.

10. Philippe Aydalot (dir.), Milieux innovateurs en Europe / Innovative environments in Europe, Paris, GREMI, 1986.

11. Statistique générale de la France, 1852, p. 334-336. 
effectif est ensuite assez stable : 14 à 15 entreprises jusqu'en $1890^{12}$. Elles sont une vingtaine à l'apogée du district vers 1920, à l'issue de la Première Guerre mondiale, durant laquelle les besoins de l'armée en chaussures ont fortement stimulé la production de cuir, notamment pour répondre au programme de la chaussure nationale voulu par le ministre Etienne Clémentel ${ }^{13}$.

Les tanneries sont la principale activité de la ville. Elles y représentent $40 \%$ des créations de sociétés entre 1890 et 1914 , et même près de $60 \%$ au début des années vingt (graphique 3$)^{14}$. Bien que de petites tailles, elles sont insérées dans des réseaux commerciaux internationaux. Elles exportent leurs cuirs en Europe centrale, en Italie, en Algérie et même beaucoup plus loin, jusqu'au Canada et en Chine ${ }^{15}$. L'influence nationale des tanneurs de Château-Renault s'exprime au sein du Syndicat général des cuirs et peaux de France, dont ils occupent un cinquième des postes de présidents et vice-présidents dans les années vingt ${ }^{16}$.

\section{Graphique 1}

\section{Évolution du nombre de tanneries à Château-Renault entre 1859 et 1939}

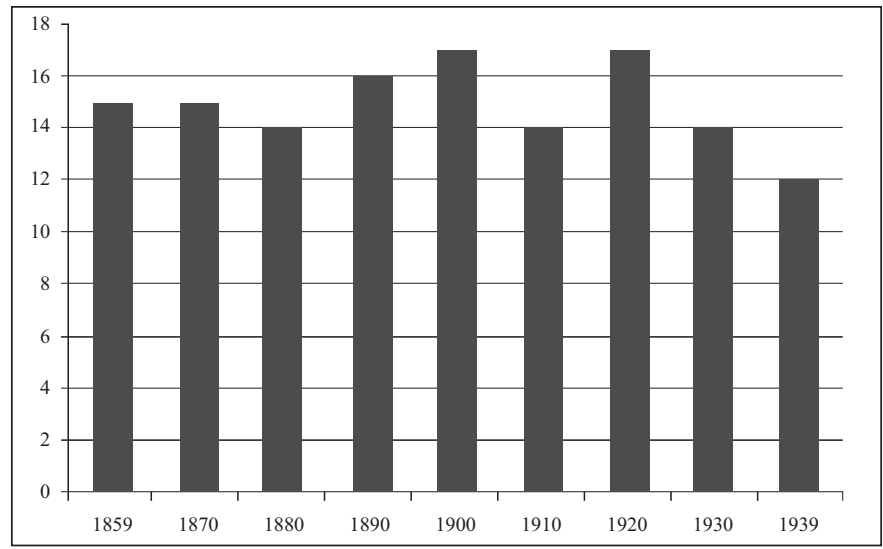

Sources : Arch. dép. 37.

12. Archives nationales (Arch. nat.) $\mathrm{F}^{12} 4508$, situation industrielle des départements, Indre-et-Loire.

13. John Godfrey, Capitalism at War: industrial policy and bureaucracy in France, 1914-1918, Hambourg, Leamington Spa, New York, Berg, 1987. Florent Le Bot, Cédric Perrin, « Mobiliser l'industrie de la chaussure, mobiliser ses territoires », Terrains et Travaux, 19, 2011.

14. Archives départementales d'Indre-et-Loire (arch. dép. 37), 4U5/68, 69 et 70, actes de sociétés, Château-Renault.

15. Arch. dép. 37, 62J49 à 57, Pelterau-Tenneson.

16. Bulletin mensuel du Syndicat général des Cuirs et Peaux de France, 220, 20 mai 1924. 
Les tanneries castelrenaudines sont des PME. La tannerie-type du district dans sa période haute (1890-1925) est une petite entreprise de 10 à 30 salariés. Elle utilise 30 à 40 fosses $^{17}$ et possède, à la fin du XIX ${ }^{\mathrm{e}}$ siècle, une chaudière à vapeur pour faire actionner des machines d'une puissance moyenne de $16 \mathrm{CV}^{18}$. Cette moyenne est cependant indicative car elle masque des écarts non négligeables et même une certaine hiérarchie. Selon les sources, le nombre d'ouvriers se situe entre 3 et 200 d'une entreprise à l'autre ${ }^{19}$. Les états nominatifs de l'Inspection du travail donnent des chiffres précis pour les années 1938-1939: 7 à 8 salariés pour les plus petits établissements (Demarle et Page, Laballe et Norgeux...), 70 à 100 pour les plus importantes (SGTF, Cuir lissé...) et même jusqu'à 205 pour l'entreprise Enault en septembre $1939^{20}$. Concernant l'outillage, les dossiers des patentes font aussi apparaître des différences sensibles. La puissance installée oscille entre 6 et $25 \mathrm{CV}$ vers $1898^{21}$, le nombre de fosses varie de 15 à 167 , et celui des marteaux à battre le cuir de 1 à 4 par entreprise.

Pour résumer, toutes sont de petites ou moyennes entreprises, mais des différences notables apparaissent entre elles, avec à un bout de l'échelle des entreprises artisanales qui emploient 3 à 5 salariés et un matériel limité (un marteau, 15 à 20 fosses) et, à l'autre bout, les " grandes maisons » du district, qui emploient jusqu'à 100 ouvriers, voire plus, et un matériel plus important (quatre marteaux, plus de 100 fosses, une ou deux machines à vapeur développant jusqu'à 25 CV vers 1900).

\section{B. DES ENTREPRISES EN RÉSEAU}

De nombreux liens unissent les destins des entreprises. Si elles sont par nature concurrentes - car elles agissent sur le même marché -, elles sont également liées par des relations de coopération et de sous-traitance. Leur correspondance montre qu'elles s'échangent aussi des informations sur les marchés d'exportation, l'arrivée de matières premières, de nouvelles techniques... La coopération est une des caractéristiques du district néo-marshalien, décrit notamment par

17. Ces fosses servent à tremper les peaux dans les jus chargés de tanins pour les transformer en cuir.

18. Les données sur les équipements proviennent des données individuelles des patentes : arch. dép. 37, 2P883, patentes. Les informations sur le niveau de production sont en revanche beaucoup plus parcellaires.

19. Dans les dossiers des patentes, il semble que tous les salariés n'étaient pas pris en compte par l'administration fiscale.

20. Arch. dép. 37, 10M256, tanneries et industries du cuir à Château-Renault.

21. L'information disparaît après cette date. 
G. Becattini ${ }^{22}$. Les factures conservées et les traites réescomptées auprès de la Banque de France montrent que les firmes travaillent les unes pour les autres ; elles se facturent des prestations entre elles. Il peut s'agir de sous-traitance de capacité - quand une tannerie s'adresse à une autre pour des cuirs qu'elle produit elle-même par ailleurs - ou de spécialité. Toutes les tanneries de la ville ne fabriquent pas exactement les mêmes produits. Les principales « places » de Château-Renault (Peltereau-Enault, Peltereau-Tenesson...) sont en capacité de maîtriser la totalité de la chaîne de production depuis l'achat des matières premières, et même la production des écorces tannantes (certaines tanneries possèdent des bois de chêne dans le nord de la Touraine et des moulins à tan), jusqu'à la commercialisation et l'exportation des cuirs finis. Les plus petites se concentrent parfois sur une étape du tannage, et notamment la dernière : le corroyage. Elles achètent alors des cuirs aux autres tanneries du district pour en assurer la finition.

Pour bien prendre la mesure du district, il faut ajouter aux tanneries les nombreuses industries annexes avec lesquelles elles sont en relation : fabriques de chaussures, brides à sabots, de colles, de moulins à tan, suif ${ }^{23}$, équarrissage, taillanderie, briqueteries et, en dehors de l'industrie proprement dite, les négoces de cuir et les banques locales. Ces activités transforment les cuirs produits dans les tanneries ou les résidus des industries, ou bien fournissent celles-ci en outils ou matières premières. Elles se développent en même temps que le district. Comme les tanneries, elles s'inscrivent dans la sphère de la petite (voire très petite) et moyenne industrie. Les fabricants de brides et d'outils sont des artisans, alors que les usines de colle et de chaussures sont constituées en société anonyme et emploient plusieurs dizaines de salariés selon les périodes, les effectifs variant beaucoup.

\section{UN BASSIN DE MAIN-D’'EUVRE FLEXIBLE}

Les tanneries emploient, selon les années, entre 500 et 700 salariés (graphique 2), dans une petite ville d'un peu plus de 4000 habitants au recensement de $1901^{24}$. Château-Renault représente, pour l'industrie du cuir, un important bassin de main-d'œuvre qualifiée, habituée au travail dans les tanneries et flexible, c'est-à-dire que les entrepreneurs emploient selon leurs besoins.

22. G. Becattini, «Dal settore industrial al distretto... », art. cit.

23. Le suif sert à faire des savons, mais aussi à la fabrication des cuirs, notamment en corroierie, pour travailler les peaux, les assouplir et les imperméabiliser.

24. Arch. nat., $\mathrm{F}^{12} 4508$, Statistique industrielle des départements, Indre-et-Loire. 
Graphique 2

Évolution du nombre de tanneries et de leurs ouvriers à Château-Renault (1869-1887)

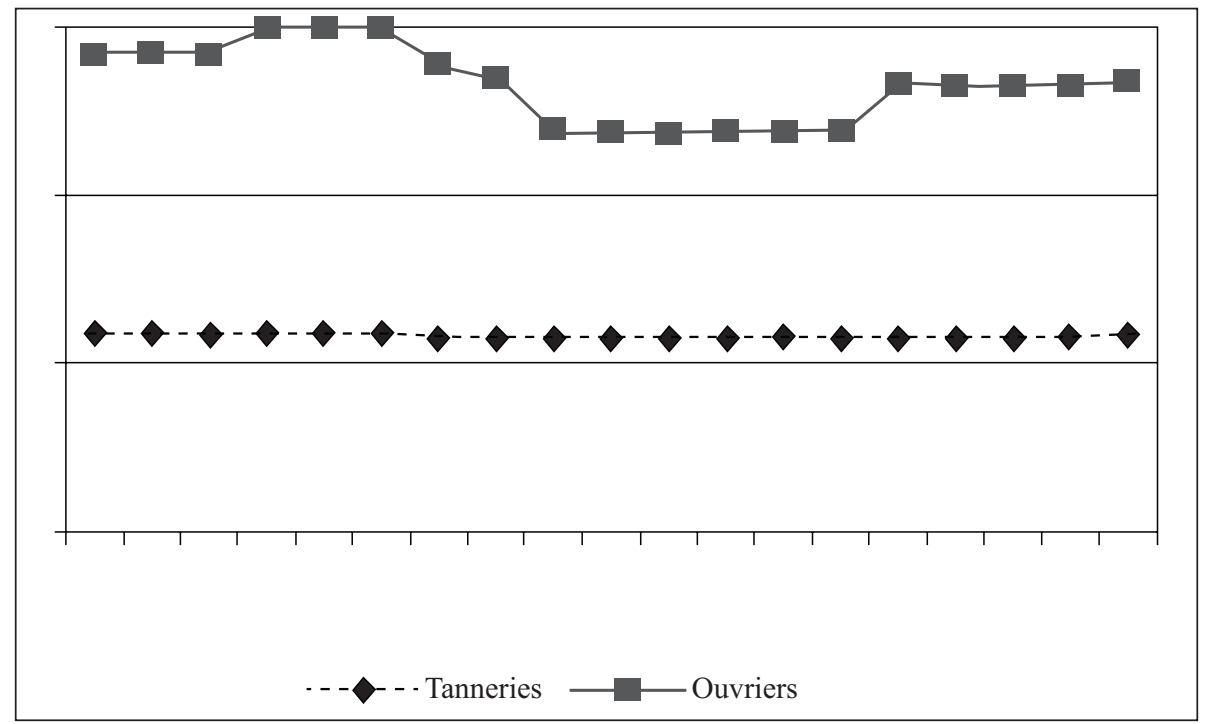

Source : Arch. nat. $\mathrm{F}^{12} 4508$.

N.B. : le graphique reprend les chiffres de la Statistique industrielle des départements pour le nombre de tanneries et celui d'ouvriers de ces établissements à Château-Renault avec une échelle logarithmique pour pouvoir comparer les deux ordres de grandeur.

Le tannage est en effet marqué par l'irrégularité de l'activité. À court terme, le travail se répartit dans l'année entre une période de forte activité et une période calme. À moyen terme, la conjoncture du secteur fait se suivre les années actives, avec de fortes ventes, et celles de ralentissement. À titre d'exemple, au cours de la période 1897-1911, les rapports de la succursale locale de la Banque de France indiquent en 1899 que « les tanneries ont réalisé pendant deux années de très gros bénéfices », mais sont « moins favorisées depuis quelques mois »; l'année suivante, elles redeviennent « particulièrement favorisées ». Le rapporteur parle de «ralentissement général des affaires » en 1901, puis de « bonne situation » en 1902. L'année 1903 voit le retour de " difficultés » qui deviennent une « crise redoutable » en 1904, tandis que 1905 et 1906 voient venir la « reprise », années suivies jusqu'en 1910 de campagnes de production « très satisfaisantes », « actives » et « prospères », avant qu'en 1911, la production diminue de nouveau. 
Face à l'instabilité, le facteur travail fait figure de volant d'ajustement. Ce caractère apparaît très clairement dans le district de Château-Renault. Les effectifs salariés sont plus élevés en été qu'en hiver et varient d'une année sur l'autre ${ }^{25}$. La saisonnalité fluidifie la gestion des variations interannuelles. Au moment de la reprise de l'activité, les entrepreneurs ajustent leurs embauches au niveau d'activité. Ils ne reprennent pas tout le monde en période creuse ou, au contraire, ils embauchent davantage que l'année précédente si les carnets de commande sont pleins. Très probablement aussi, cela permet de ne pas réembaucher au printemps les éléments qui se seraient montrés trop revendicatifs. Le graphique 2 montre bien que si le nombre d'entreprises reste stable malgré les aléas du marché, celui des effectifs ouvriers varie beaucoup. Ce sont donc ces derniers qui supportent l'essentiel du poids des variations.

Les employeurs imposent à leurs ouvriers une grande flexibilité, synonyme de chômage. Cette caractéristique du marché de l'emploi se retrouve dans les centres tannants du sud de la France. Interpellant le ministre du Travail René Viviani, le 17 janvier 1910, au sujet des grévistes de Graulhet, Jean Jaurès parle « de chômage périodique, de chômages annuels toujours plus graves ${ }^{26}$ ».

\section{LE DISTRICT SE FRAGILISE}

\section{A. D'UN SAVOIR-FAIRE EN COMMUN AU CONSERVATISME TECHNIQUE : L'ATTACHEMENT AU TANNAGE VÉGÉTAL}

La renommée nationale, et même internationale, des cuirs de Château-Renault repose en particulier sur la maîtrise, par ses tanneurs, de la technique de production : le tannage extralent aux écorces végétales. Elle se vérifie dans les tentatives de concurrents d'autres régions d'utiliser des appellations commerciales ambiguës qui évoquent la Touraine. Les tanneurs castelrenaudins en ont bien conscience et décident de s'unir pour défendre ce qu'ils considèrent comme un patrimoine commun. Ce front commun pour la défense du produit se réalise autour de la marque déposée auprès du tribunal de commerce de Tours, en deux étapes : le 27 novembre 1886, puis le 29 août $1891^{27}$. Dans le préambule de la convention qui régit l'usage de la marque, les « fabricants

25. Arch. dép. 37, 2P883, patentes.

26. Cité dans François Jarrige, « Une invention de Jaurès ? La grève de Graulhet entre hygiénisme et machinisme au début du XX⿳亠丷厂 siècle ", Cahiers Jaurès, 199, 2011, p. 9-25.

27. Arch. dép. 37, 6U241, registre des dépôts des marques de fabrique. 
tanneurs » de la ville se disent « désireux de sauvegarder leurs intérêts industriels et de défendre la réputation des cuirs de Château-Renault, réputation qui est la propriété collective de tous les fabricants de cette ville ${ }^{28} »$. La marque consiste notamment dans les lettres UT (pour Union des tanneurs) et la mention « sans surcharge » (comprendre de tannins et donc un tannage lent). Elle ne peut être appliquée que sur les cuirs entièrement fabriqués à Château-Renault, selon les conditions prévues par la convention. À l'image de quelques autres districts, celui de la porcelaine de Limoges par exemple, Château-Renault se dote d'une marque collective ${ }^{29}$. La démarche est significative pour au moins trois raisons. Tout d'abord, elle témoigne d'un certain consensus technique parmi les tanneurs du lieu, et cet aspect renvoie à la circulation interne des techniques, qui sont « comme dans l'air», selon l'expression d'Alfred Marshall. Ensuite, le choix de s'unir plutôt que d'agir individuellement sur le marché entre typiquement dans le schéma de la coopération/concurrence (ou coopétition) qui fait l'originalité des districts. Enfin, la marque collective est déposée, puis gérée et défendue par le syndicat professionnel local, l'Union des tanneurs de Château-Renault, qui est un exemple d'une institution de district ${ }^{30}$.

Cependant, le tannage végétal lent qui a fait la force et la renommée du district à la fin du XIX ${ }^{\mathrm{e}}$ et au début du XX $\mathrm{X}^{\mathrm{e}}$ siècle devient une faiblesse et un handicap au $\mathrm{XX}^{\mathrm{e}}$ siècle. L'industrie vit alors une rupture technique avec l'apparition, puis la diffusion rapide d'une nouvelle méthode, le tannage minéral au chrome. Cette évolution majeure s'inscrit dans la continuité de la recherche de procédé de tannage plus rapide du siècle précédent. Mais les tanneurs de Château-Renault tournent délibérément le dos à cette innovation et restent viscéralement attachés au tannage végétal lent sur l'exploitation duquel ils ont construit leur succès. La résistance du tannage végétal n'est pas tout à fait spécifique à ChâteauRenault. Cette inertie technique se retrouve ailleurs en France. Ce qui paraît plus original, c'est la non-pénétration et même le refus du tannage au chrome à Château-Renault. En Normandie, par exemple, les méthodes végétales et minérales ont coexisté au moins jusqu'en $1940^{31}$.

À Château-Renault, le tannage au chrome ne s'implante pas. Deux exemples, pris parmi d'autres, viennent le démontrer. Il existe bien un cas au moins d'une

28. Idem.

29. Florent Le Bot, «Limoges Unique France» (1928-1938). Le patronat collectif de la porcelaine et la défense ou l'illustration d'un produit spécifique », in C. Druelle-Korn (dir.), Les corps intermédiaires économiques. Entre l'Etat et le marché, Limoges, PULIM, 2011, p. 175.

30. Florent Le Bot et Cédric Perrin, «Brands and collective identity. The case of french industrial districts ", in les actes du congrès de l'Associación española de historia económica, Madrid, 2014, à paraître.

31. Philippe Ponsot, Tanneries et tanneurs du Cinglais 1746-1962, Cully, OREP Éditions, 2005. 
tannerie castelrenaudine qui s'essaie précocement au tannage au chrome. Mais sa tentative est un échec. Elle est une des premières à disparaître dans les années vingt. Cette déconvenue a sans doute conforté les réticences des autres tanneurs. L'autre exemple est celui d'un tanneur de Loire-Inférieure, qui veut installer une tannerie au chrome à Château-Renault en $1914^{32}$. Alors que jusqu'alors, la préfecture a donné son accord à toutes les demandes, lui se voit opposer un refus. Les tanneurs de la ville se sont dressés contre le projet et le préfet s'est rallié à leur opinion. Ainsi, le seul cas (connu) de refus à Château-Renault est lié au tannage au chrome.

À la fin du $\mathrm{XX}^{\mathrm{e}}$ siècle (alors que le district tourangeau a déjà disparu), le tannage végétal bénéficie d'un renouveau en se parant des vertus du développement durable ${ }^{33}$. Aux XIX ${ }^{\mathrm{e}}$ et $\mathrm{XX}^{\mathrm{e}}$ siècles, il apparaît pourtant comme une activité polluante. La préfecture d'Indre-et-Loire reçoit régulièrement des plaintes nombreuses à l'encontre des nuisances créées par les tanneries, particulièrement la pollution des eaux de la Brenne ${ }^{34}$. L'ancrage territorial du district apparaît affaibli, tout du moins en partie, par les contestations et les conflits que celui-ci suscite en son sein et dans ses environs.

\section{B. LES PROBLÈMES FINANCIERS}

Les tanneries sont sans cesse confrontées à des problèmes de trésorerie, qui sont en grande partie dus au choix technique du tannage végétal, ou au moins accentués par lui. Le tannage végétal est en effet un tannage lent. Les tanneurs parlent même de tannage extralent. Il faut 18 mois, parfois plus, pour transformer une peau brute (dite « verte ») en cuir. La méthode permet de produire les cuirs de grande qualité qui ont fait la réputation de la ville et les tanneurs y sont fermement attachés. Mais elle suscite de lourdes immobilisations financières. Le fabricant tanneur doit attendre de longs mois entre le moment où il achète sa matière première et celui où il la revend en cuir tanné et en tire un bénéfice. Dans l'intervalle, son capital est immobilisé dans les fosses de tannage. En outre, l'habitude de payer à terme $(30,60$ ou 90 jours) rallonge encore le délai avant les premiers bénéfices. Autrement dit, le capital circulant circule lentement. Les rapports de la Banque de France évoquent régulièrement les problèmes de trésorerie dans les tanneries castelrenaudines. Aussi les banques

32. Arch. dép. 37, 5M255, établissements classés, dossier Viéron.

33. Cédric Perrin, « Le développement durable en perspective historique : l'exemple des tanneries », in O. Dard, C. Didry, F. Le Bot, C. Perrin, Les mille peaux du capitalisme, numéro spécial de L'Homme et la Société, 2, 2014.

34. Arch. dép. 37, 5M195, pollution de la Brenne. 
locales jouent-elles un rôle important dans le financement des activités, notamment commerciales, du district. Entre fournisseurs et clients, les entreprises tirent des traites qui sont autant de reconnaissances de créances commerciales. Pour financer sa trésorerie, l'entreprise créancière va présenter sa traite auprès de sa banque, qui lui prête le montant de la traite moins les intérêts : c'est l'escompte. Les banques elles-mêmes peuvent se refinancer en présentant les papiers commerciaux auprès de la Banque de France, qui fait office de prêteur en dernier ressort. Jusqu'au début du $\mathrm{XX}^{\mathrm{e}}$ siècle, ce système accorde un rôle central aux banques locales et régionales ${ }^{35}$. Les tanneries sont de petites entreprises et trouvent plus facilement à se financer auprès de ces banques. La proximité fait la confiance qui fait le prêt. De ce point de vue, le district industriel est un atout grâce à l' ' atmosphère » industrielle qui le caractérise, selon l'économiste anglais Alfred Marshall ${ }^{36}$. Le district a ses banques locales : les banques Baugé et Diard. L'essentiel de leur clientèle est composé des tanneries de la ville. La succursale de Tours de la Banque de France ouvre une antenne à Château-Renault en 1901. Ces établissements sont également des institutions du district ; ils contribuent fortement à l'organiser. Mais la progressive disparition des banques locales au début du XX $\mathrm{X}^{\mathrm{e}}$ siècle fragilise les PME de la tannerie. $\mathrm{La}$ banque Baugé, par exemple, disparaît en $1907^{37}$.

\section{L'AFFAIBLISSEMENT DU TERRITOIRE}

Alors même que le district est à son apogée, ses entreprises se trouvent de plus en plus intégrées dans des réseaux à l'échelle nationale, voire internationale, qui les éloignent du territoire. Déjà, ce dernier ne fournit plus qu'une petite partie des matières travaillées dans les usines castelrenaudines. Les tanneries possèdent des bois de chêne dans le nord de la Touraine, des moulins à tans..., mais une grande part des écorces tannantes est achetée ailleurs et arrive par train à la gare de Château-Renault. Le même schéma se répète avec les peaux. Une partie provient des élevages bovins locaux. Des abattoirs et des équarrissages comptent parmi les activités annexes du district. Au niveau national, les syndicats professionnels, dans lesquels les patrons tanneurs de Château-Renault

35. Michel Lescure, « Entre villes et campagne : l'organisation bancaire des districts industriels. L'exemple du Choletais (1900-1950) », in J.-F. Eck, M. Lescure (dir.), Villes et districts industriels en Europe occidentale XVII ${ }^{e}$-XX ${ }^{e}$ siècles, Tours, PUFR, 2002, p. 81-102. Florent Le Bot, « La chute de la banque Beaucé et $C^{\text {ie }}$. La remise en cause de l'équilibre fragile du système industriel fougerais durant les années 1930 », Annales de Bretagne et des pays de l'Ouest, 114, 2007, p. 75-88.

36. Alfred Marshall, Principles of Economics, op. cit.

37. Banque de France, rapport de la succursale de Tours, 1908. 
sont bien représentés, militent d'ailleurs pour faire passer auprès des éleveurs des consignes qui garantissent la qualité des peaux locales (comme l'abandon de l'aiguillon ou des barbelés, qui abîment les peaux $)^{38}$. Il n'en reste pas moins que les élevages locaux ne suffisent pas à approvisionner les tanneries qui doivent acheter des peaux vertes sur un marché plus vaste, et même en importer, notamment d'Amérique du Sud.

Surtout, s'agissant de la cohésion et de l'ancrage territorial du district, les tanneries de Château-Renault se trouvent prises dans des mouvements de restructuration du secteur à l'échelle nationale. Dans un contexte global de diminution du nombre d'entreprises ${ }^{39}$, l'industrie est transformée par une série de fusions. Les tanneries tourangelles ne restent pas à l'écart. Elles se rapprochent d'autres acteurs du secteur installés dans d'autres villes. Les « grandes » entreprises de Château-Renault en particulier se rapprochent de tanneries de la région parisienne. De nouvelles sociétés sont fondées à cette occasion. Les dénominations changent. La vieille maison Peltereau fusionne avec une autre de Montrouge et devient les Établissements Enault en 1917. L'entreprise Brossillon-Delamotte devient la Société générale des tanneries françaises (SGTF) en 1918. À l'occasion de ces transformations, le siège social est implanté en région parisienne, ou ailleurs : l'entreprise Roux, qui rachète la tannerie Nioré-Bienvenu en 1918, est installée à Romans, en Ardèche, l'un des districts français de la chaussure ${ }^{40}$. Il n'est pas anodin non plus que les propriétaires des " grandes tanneries » vivent désormais à Paris et ne conservent plus qu'un pied-à-terre en Touraine. La dynamique éloigne les centres de décision. Château-Renault devient un territoire usinier qui n'a plus son destin en main. Pour Ann Markusen, dans un district marshallien, les entreprises sont possédées par des patrons locaux qui décident leurs investissements et leurs productions localement, et la coopération avec des firmes extérieures au district est minimale ${ }^{41}$. Ce n'est donc plus tout à fait vrai dans le cas de Château-Renault.

\section{LE LENT DÉCLIN DU DISTRICT}

Si à la fin des années dix et au début des années vingt, le district de ChâteauRenault semble être à son apogée - le nombre de tanneries dans la ville est

38. Arch. nat., $\mathrm{F}^{12}$ 8807, commission consultative des cuirs et pelleteries, mai 1923.

39. Cf. infra, $3^{\mathrm{e}}$ partie.

40. Cf. F. Le Bot, C. Perrin, « Mobiliser l'industrie ...», op. cit.

41. A. Markusen, « Sticky places in Slippery space ...», op. cit., p. 299. 
alors au plus haut -, ses fondements sont fragilisés et la dynamique ne tarde pas à se retourner.

Dès les années vingt, plusieurs indicateurs passent au rouge. Déjà, la population de la ville recule : 4097 habitants en 1926 contre 4299 en 1906. Le chiffre remonte légèrement à 4106 en 1931 avant de retomber à 3877 en 1936 sous l'effet de la crise économique des années trente. Alors qu'elle est la principale activité de la ville, l'industrie du cuir ne paraît plus assez solide pour retenir les habitants, qui préfèrent aller chercher du travail ailleurs. Le bassin d'emploi se rétracte. Les années vingt sont aussi marquées par un retournement de la démographie des entreprises. Les créations de nouvelles sociétés de tannerie s'interrompent brutalement. Elles ne sont plus majoritaires dans le total des créations de sociétés (graphique 3). Les tanneries cessent d'être motrices dans l'activité économique de la ville. 


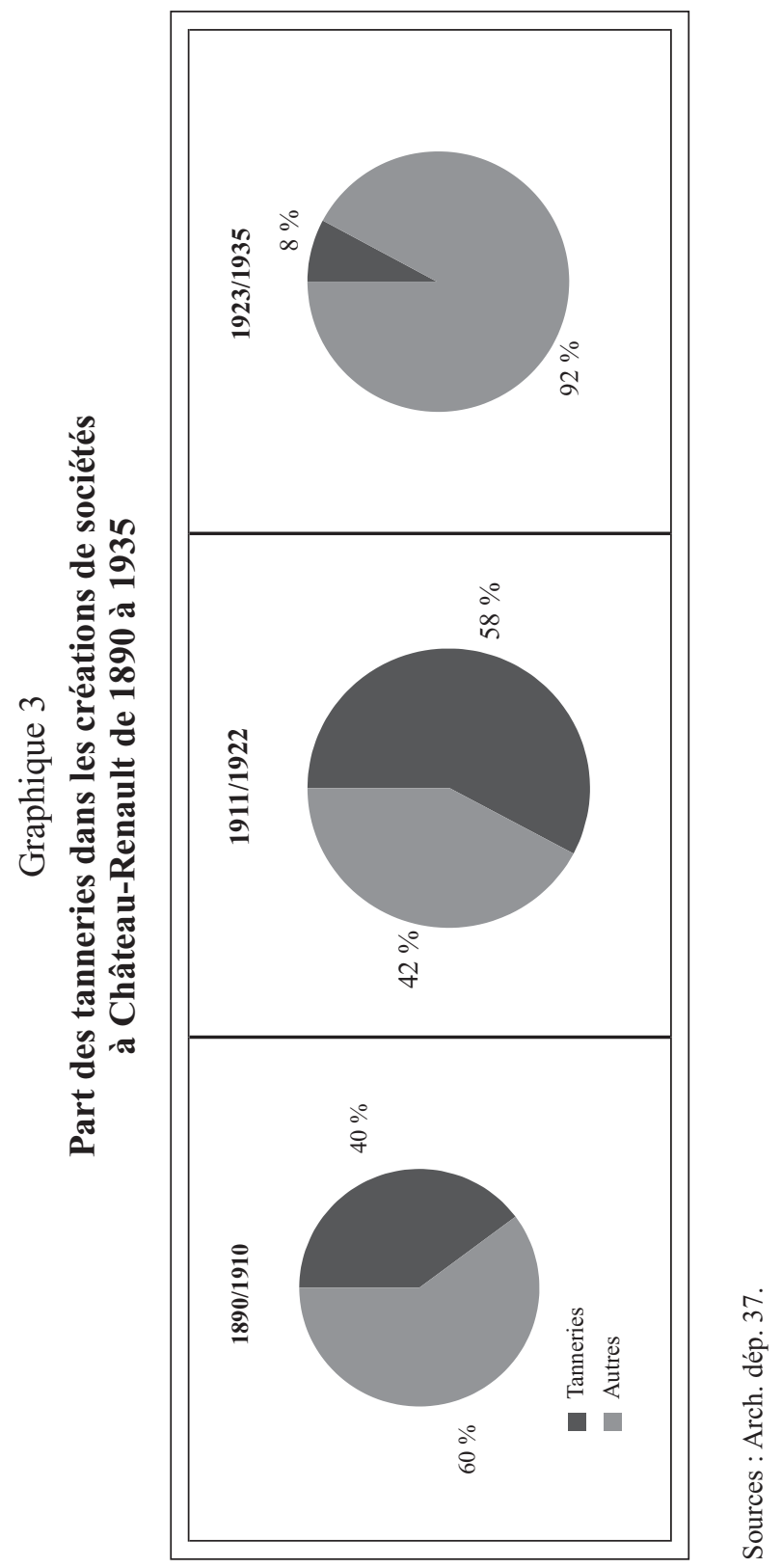


Faute de se renouveler, le parc d'entreprises vieillit rapidement et se contracte. Beaucoup de créations de l'immédiat après-guerre s'avèrent éphémères. Mais de plus anciennes et importantes tanneries ferment aussi sans être remplacées. L'union de Nioré-Bienvenu avec les Établissements Roux se termine par la fermeture de l'usine de Château-Renault en 1927, Roux poursuivant ses activités à Romans ${ }^{42}$. La crise des années trente ne fait que renforcer les problèmes. De nouvelles entreprises disparaissent (graphique 4). Les tanneries ne sont plus qu'une douzaine à la fin des années trente.

Graphique 4

\section{Évolution du nombre de tanneries à Château-Renault de 1920 à 1985}

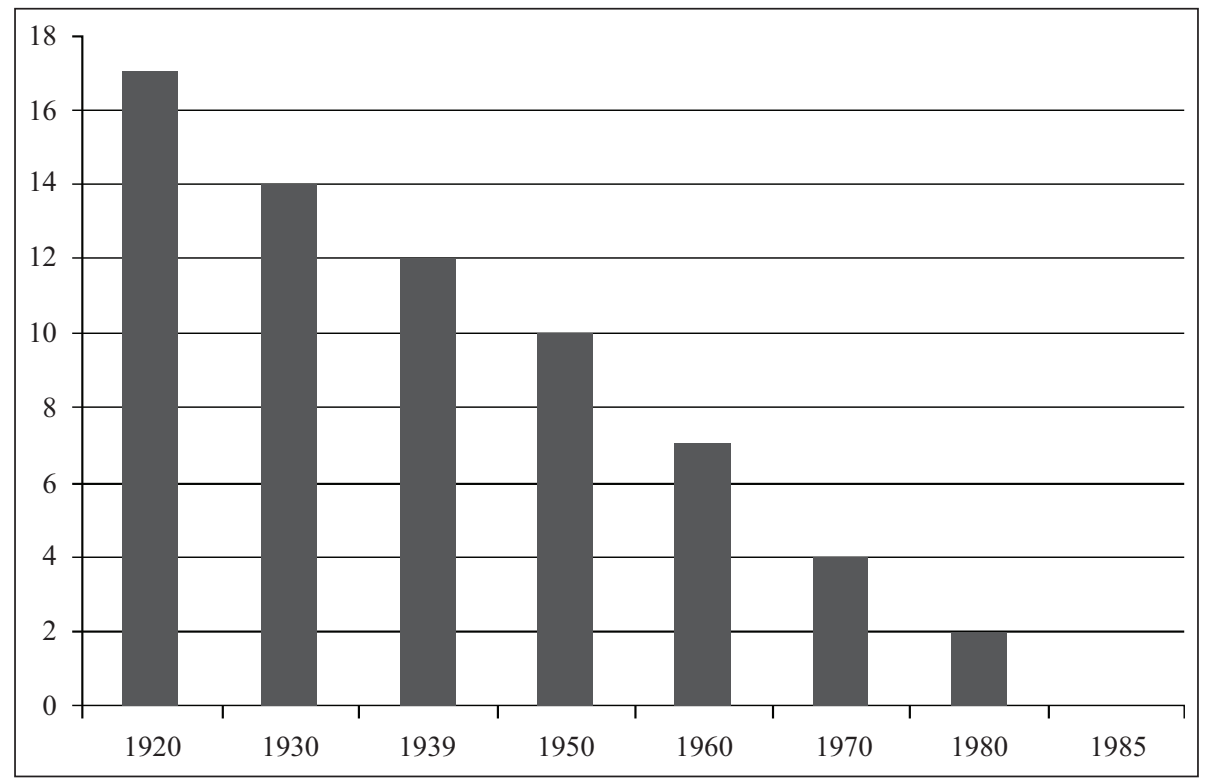

Source : Arch. dép. 37.

Plusieurs facteurs expliquent les difficultés, parmi lesquels deux niveaux se dégagent : un général et un autre interne au district. Il faut bien voir que la fragilisation du district tourangeau se situe dans un contexte de difficultés

42. Arch. dép. 37, 4U5/69 Actes de sociétés de Château-Renault. 
générales de l'industrie du cuir et de la tannerie. Depuis le début du XX $\mathrm{X}^{\mathrm{e}}$ siècle, la filière cuir perd des entreprises et des emplois ${ }^{43}$. Alors que l'ensemble des tanneries, corroieries et mégisseries employaient près de 40000 salariés en France au début du XX $X^{\mathrm{e}}$ siècle, elles n'en emploient plus que 23000 au milieu des années cinquante, soit presque moitié moins. Les métiers du cuir sont confrontés à un problème de débouchés. Les usages du cuir diminuent, donc la demande. La production de chaussures, grande consommatrice, était le principal débouché des grands cuirs tannés à Château-Renault. Or cette industrie aussi est en déclin ${ }^{44}$. Situé en amont de la filière, le tannage subit les répercussions des problèmes de tous les autres chaînons.

Mais les difficultés du district sont également la conséquence des choix de ses entrepreneurs. À cet égard, relevons trois points principaux. Le premier est que les produits de grande qualité sortis des usines de Château-Renault ont du mal à s'écouler, en particulier pendant la crise des années trente ${ }^{45}$. Les rapports de la Banque de France signalent que les prix sont trop élevés. Dans les années cinquante, une brochure du Syndicat des tanneurs d'Indre-et-Loire met encore en avant « la qualité des cuirs », associée à « une technique excellente ${ }^{46}$ ». Les producteurs de Château-Renault sont restés fidèles à la technique du tannage végétal, qui produit des cuirs de qualité supérieure, alors que le marché est en attente de productions plus courantes et moins chères. Le deuxième point, corrélatif, est que les problèmes de trésorerie s'accentuent au point d'acculer certaines entreprises à cesser leur activité. En troisième point, enfin, le district commence à subir les conséquences négatives du développement des coopérations et des relations avec l'extérieur, qui brouillent ses réseaux internes. Dans le cas de Roux et de Château-Renault, par exemple, la logique de groupe prévaut sur celle du territoire et aboutit à une décision défavorable au district : la fermeture de l'usine de Château-Renault. Pour autant, l'échec du district n'est pas encore acté dès la fin des années trente. D'une part, il jouit encore de son prestige et d'une certaine attractivité. En 1937, un tanneur du Nord vient s'y installer. D'autre part, si le nombre d'entreprises diminue, quelques-unes emploient plus d'ouvriers en 1938-1939. Il y a donc un mouvement de concentration.

Les difficultés croissent après la guerre. La situation s'aggrave : la moitié des tanneries disparaît entre 1947 et 1957. Pourtant, dans les années cinquante, plusieurs tanneurs tentent de relancer la coopération interfirmes caractéristique du district. Mais leur tentative échoue, du fait notamment de la disparition pro-

43. F. Le Bot, La fabrique réactionnaire..., op. cit.

44. F. Le Bot, La fabrique réactionnaire..., op. cit.

45. F. Le Bot, C. Perrin, " Brands and collective identity... », art. cit.

46. Arch. dép. 37, 48J26, Syndicats des cuirs et peaux de Touraine, juillet 1955. 
gressive des parties prenantes. Dans une lettre adressée à Michel Debré, sénateur d'Indre-et-Loire, le 11 mars 1953, le directeur des Établissements Enault parle de «marasme » des industries de la tannerie ${ }^{47}$. Son entreprise ferme l'année suivante. C'était la plus importante du lieu en nombre d'employés. On assiste dans le même temps à une remobilisation du territoire grâce à la décentralisation industrielle. Relativement proche de Paris, bien desservi par le réseau routier, le site de Château-Renault intéresse les candidats à la décentralisation : 9 des 24 dossiers soutenus par le Fonds de développement économique et social dans le département de l'Indre-et-Loire concernent Château-Renault ${ }^{48}$. La ville regagne des habitants : 4238 en 1962, 6043 en 1975. Toutefois, le mouvement ne concerne que très marginalement les tanneries. Seul Mersch est intéressé, mais il ne reste que quelques années avant de repartir ${ }^{49}$. Quand la décentralisation industrielle vient donner un nouveau souffle à l'activité industrielle locale, il ne reste déjà plus grand-chose du district : 7 tanneries en 1960, 2 en 1980 (graphique 4). La dernière ferme en 1985.

*

$* *$

Les districts naissent, se développent, périclitent et certains disparaissent. Aujourd'hui, de nombreux districts sont sur le déclin - pour ne citer que quelques exemples en France : Fougères, Romans, le Choletais... En Italie même, le nombre de districts recensés officiellement par l'ISTAT a baissé de 199 à 156 entre 1991 et 2001, et certains observateurs, comme Luciano Gallino, envisagent la disparition de la troisième Italie ${ }^{50}$. Le district est confronté à un nombre croissant d'échecs. Le cas de Château-Renault est à resituer dans cette perspective et offre quelques éléments d'interprétation. Il s'agit certes d'un district de petite taille et il serait tentant de penser qu'il n'avait pas atteint la masse critique qui lui aurait permis de résister et durer, mais des systèmes productifs locaux plus importants n'ont pas mieux survécu. En réalité, l'exemple de Château-Renault montre bien que le district a été une organisation adaptée et efficace à un moment donné - ici la fin du XIX et le début du XX $X^{\mathrm{e}}$ siècle -, mais qu'il n'a pas permis de suivre les reconfigurations du système productif

47. Arch. dép. 37, 48J29, correspondances des tanneries.

48. Arch. nat., $\mathrm{F}^{12} 11331$ à 11334 .

49. Arch. dép. 37, 5M258, courrier des Établissements Mersch au préfet d'Indre-et-Loire du 9 mai 1962. Sur J. Mersch, voir sa notice dans Jean-Claude Daumas, Alain Chatriot, Danièle Fraboulet, Patrick Fridenson et Hervé Joly (dir.), Dictionnaire historique des patrons français, Paris, Flammarion, 2011.

50. Andrea Colli, « Modelos de empresas familiares en Italia, 1980-2005. De los mercados locales a los globales », in Jordi Catalan, José Antonio Miranda, Ramon Ramon-Muñoz (dir.), Distritos y clusters..., op. cit., p. 360. Luciano Gallino, La scomparsa dell'Italia industriale, Turin, Einaudi, 2003. 
à l'œuvre dans cette branche d'activité industrielle. Pris dans un mouvement de concentration et de fusion à une autre échelle, les établissements du district perdent ce qui était leur force, leur ancrage territorial et leur capacité endogène de décision. Le territoire ne paraît plus suffire à fournir à ses entreprises les ressources dont elles ont besoin. Elles s'en éloignent, le district perd de sa cohérence et finit par disparaître. Mais les ressources du territoire ne sont pas seules, ni même principalement, en cause : elles n'agissent pas elles-mêmes. Ce sont bien les décisions des entrepreneurs du district qui pèsent de façon déterminante sur son évolution. Ainsi, le choix de conserver la technique du tannage végétal lent a aggravé les problèmes de débouchés commerciaux et les difficultés financières des tanneries castelrenaudines. Ce sont les entrepreneurs qui se sont éloignés du district et l'ont mis en échec. 